\title{
Oxidative-reductive photodecomposition of perfluorooctanoic acid in water
}

\author{
R. R. Giri • H. Ozaki $\cdot$ X. Guo $\cdot$ R. Takanami $\cdot$ \\ S. Taniguchi
}

Received: 9 January 2013/Revised: 1 March 2013/Accepted: 23 April 2013/Published online: 25 May 2013

(C) Islamic Azad University (IAU) 2013

\begin{abstract}
This article aims to elucidate on usefulness of vacuum ultraviolet (VUV) for photoreductive degradation of perfluorooctanoic acid (PFOA), a representative perfluorinated compound (PFC), in water for the first time. Bench-scale tests were conducted on oxidative and reductive (with aquated electron: $e_{\mathrm{aq}}^{-}$) mineralization of PFOA using low-pressure UV (LPUV) lamps and potassium iodide. Unlike with $254 \mathrm{~nm}$ wavelength (UVC), the reductive mineralization with VUV was very inefficient compared to the corresponding oxidative mineralization. The inefficiency is attributed to low reactivity of $e_{\mathrm{aq}}^{-}$with PFOA and its fluorinated products than that of $185 \mathrm{~nm}$ photons. Direct VUV photolysis of PFOA and its products in reductive reaction conditions was not apparent due to a very big difference in reactivity of 185 and $254 \mathrm{~nm}$ photons with iodide. The results demonstrated that highly energetic VUV photons are not suitable for photoreductive degradations of PFCs involving $e_{\mathrm{aq}}^{-}$, but they can be best used for oxidative degradations. These findings should serve as an important reference on VUV usage to decompose refractory micropollutants.
\end{abstract}

R. R. Giri $(\bowtie)$

New Industrial R \& D Center, Osaka Sangyo University, 3-1-1 Nakagaito, Daito City, Osaka 574-8530, Japan

e-mail: rabindra@cnt.osaka-sandai.ac.jp

H. Ozaki $\cdot$ R. Takanami $\cdot$ S. Taniguchi

Department of Civil Engineering, Osaka Sangyo University,

3-1-1 Nakagaito, Daito City, Osaka 574-8530, Japan

X. Guo

Graduate School of Engineering, Osaka Sangyo University, 3-1-1 Nakagaito, Daito City, Osaka 574-8530, Japan
Keywords Aquated electrons - Defluorination · Mineralization · Oxidation · Reduction · Vacuum ultraviolet

\section{Introduction}

Occurrence of perfluorinated compounds (PFCs) in water environment and their negative impacts on aquatic ecosystem and human health has been a major focal point of researchers and scientists particularly in the industrialized countries for the last several years. Among the PFCs, perfluorooctanoic acid (PFOA) and perfluorooctane sulfonate (PFOS) have received more attentions as the compounds are widely detected particularly in surface water bodies (Saito et al. 2004; Lien et al. 2006). Effluents from wastewater treatment plants (domestic as well as industrial) are pointed out as the major point sources of these compounds to the water bodies (Clara et al. 2008). Moreover, increasing number of published research reports suggests potential negative impacts of PFCs in water environment on aquatic life and human health (Lau et al. 2004; Corsini et al. 2012; Domingo 2012).

The PFCs are very stable against oxidation, while this characteristic is attributed to the highest electronegativity of fluorine atom $(\approx 4.0)$ and very strong $\mathrm{C}-\mathrm{F}$ bond $(\approx 552 \mathrm{~kJ} / \mathrm{mol})$. The advanced oxidation techniques (AOTs) also cannot eliminate the compounds in water due to the inability of powerful and non-selective hydroxyl radicals $\left({ }^{\circ} \mathrm{OH}\right)$ to oxidize them. However, ultraviolet (UV) radiation-based oxidation methods are shown to be more promising than other AOTs. Although direct UVC (254 nm) photolytic degradation of PFOA is negligibly small (Chen et al. 2007; Giri et al. 2011), use of chemical oxidants together with UVC can decompose the compound 
more easily (Chen and Zhang 2006; Wang et al. 2008; Cao et al. 2010; Tang et al. 2012). Furthermore, it has been demonstrated during the last few years that vacuum UV (VUV) $(185 \mathrm{~nm})$ photolysis is more efficient than other VU-based methods for PFOA degradation (Chen et al. 2007; Giri et al. 2011, 2012). The carbon-carbon bond in carboxyl functional group is cleaved first followed by formation of fluoride ions due to hydrolysis in stepwise manner during UV oxidation of PFOA and other shorter carbon-chain perfluoroalkyl carboxylates as illustrated in the following equations (Chen et al. 2007):

$$
\begin{aligned}
& \mathrm{C}_{7} \mathrm{~F}_{15} \mathrm{COOH}(\mathrm{PFOA})+\mathrm{h} v \rightarrow \mathrm{C}_{7} \mathrm{~F}_{15} \cdot+\cdot \mathrm{COOH} \\
& \mathrm{C}_{7} \mathrm{~F}_{15} \cdot+\mathrm{H}_{2} \mathrm{O} \rightarrow \mathrm{C}_{6} \mathrm{~F}_{13} \mathrm{COOH}(\mathrm{PFHpA})+\mathrm{F}^{-} \\
& \mathrm{C}_{6} \mathrm{~F}_{13} \mathrm{COOH}(\mathrm{PFHpA})+\mathrm{h} v \rightarrow \mathrm{C}_{6} \mathrm{~F}_{13} \cdot+\cdot \mathrm{COOH} \\
& \mathrm{C}_{6} \mathrm{~F}_{13} \cdot+\mathrm{H}_{2} \mathrm{O} \rightarrow \mathrm{C}_{5} \mathrm{~F}_{11} \mathrm{COOH}(\mathrm{PFHxA})+\mathrm{F}^{-}
\end{aligned}
$$

Recently, photoreductive reaction using UVC and potassium iodide (KI) is shown as an efficient method than direct UVC photolysis for PFOA degradation and defluorination in water (Park et al. 2009; Park 2010; Qu et al. 2010). Reaction between UVC and KI in absence of dissolved oxygen (DO) generates aquated electrons $\left(e_{\mathrm{aq}}^{-}\right)$ (Iglev et al. 2005), which is a powerful reductant $\left(E_{\mathrm{aq} / e}{ }^{\circ}=\right.$ $-2.9 \mathrm{~V}$ ) responsible for the enhanced PFOA degradation and defluorination. Unlike in UV photooxidation, carbonfluorine bonds are cleaved directly by $e_{\mathrm{aq}}^{-}$as nucleophile during photoreductive degradation of PFOA and other shorter carbon-chain perfluoroalkyl carboxylates as illustrated in the following equations (Qu et al. 2010):

$\mathrm{I}^{-}+\mathrm{h} v \rightarrow \mathrm{I}+e_{\mathrm{aq}}^{-}$

$\mathrm{C}_{7} \mathrm{~F}_{15} \mathrm{COOH}(\mathrm{PFOA})+e_{\mathrm{aq}}^{-} \rightarrow \mathrm{C}_{7} \mathrm{~F}_{14} \mathrm{HCOOH}+\mathrm{F}^{-}$

$\mathrm{C}_{7} \mathrm{~F}_{14} \mathrm{HCOOH}+e_{\mathrm{aq}}^{-} \rightarrow \mathrm{C}_{6} \mathrm{~F}_{13} \mathrm{COOH}(\mathrm{PFHpA})+\mathrm{F}^{-}$

$\mathrm{C}_{6} \mathrm{~F}_{13} \mathrm{COOH}(\mathrm{PFHpA})+e_{\mathrm{aq}}^{-} \rightarrow \mathrm{C}_{6} \mathrm{~F}_{12} \mathrm{HCOOH}+\mathrm{F}^{-}$

$\mathrm{C}_{6} \mathrm{~F}_{12} \mathrm{HCOOH}+e_{\mathrm{aq}}^{-} \rightarrow \mathrm{C}_{5} \mathrm{~F}_{11} \mathrm{COOH}(\mathrm{PFHxA})+\mathrm{F}^{-}$

Nevertheless, usefulness of the method has yet to be fully understood. Furthermore, it is not yet known what would happen with PFOA degradation and defluorination efficiencies if VUV $(185 \mathrm{~nm})$ is used in photoreductive reaction conditions. The first-order rate value for photoreductive degradation of PFOA with aquated electrons $\left(7.3 \times 10^{-3} \mathrm{~min}^{-1}\right.$; Qu et al. 2010) and a roughly estimated first-order PFOA photolysis rate value $\left(10.2 \times 10^{-3} \mathrm{~min}^{-1}\right.$; Giri et al. 2011, 2012) with VUV $(185 \mathrm{~nm})$ indicate that photoreductive degradation of PFOA with VUV could be significantly slower than its VUV photolysis degradation. However, this point has not yet been experimentally verified. Moreover, it is also not known whether photolysis and photoreductive reactions can occur simultaneously in reductive reaction conditions using $\mathrm{KI}$ when both the wavelengths (254 and $185 \mathrm{~nm}$ ) from lowpressure UV (LPUV) lamps are transmitted to reaction solution.

This article aims to (1) clarify whether photoreductive degradation of PFOA in water with VUV is really a slow reaction process than its VUV photolysis and (2) assess whether both photolysis and photoreductive reactions occur simultaneously in reductive reaction conditions utilizing $\mathrm{KI}$ in presence of both UVC and VUV. Simple laboratory tests were conducted on UV photolytic and photoreductive degradations of PFOA in pure water using two LPUV lamps and KI in the laboratory of New Industrial Research and Development Center, Osaka Sangyo University, Japan, during May to October of 2012.

\section{Materials and methods}

Standards and reagents

Perfluorooctanoic acid (PFOA) standard (CAS: 335-67-1, $99 \%$ purity, Fluorochem Ltd.) was supplied by Wako Pure Chemical Industries Ltd., Osaka. PFOA internal standard $\left({ }^{13} \mathrm{C}_{4}\right.$-PFOA, catalog No: MPFOA) was obtained from Wellington Laboratories, Canada. Standards for perfluoroheptanoic acid (PFHpA), perfluorohexanoic acid (PFHxA), perfluoropentanoic acid (PFPeA) and perfluorobutanoic acid (PFBA) were obtained from Wellington Laboratories, while those for perfluoropropionic acid (PFPrA) and trifluoroacetic acid (TFA) were supplied by Wako Pure Chemical Industries Ltd. Other necessary chemicals like acetonitrile (LC/MS grade), ammonium formate, KI were also supplied by Wako Pure Chemical Industries Ltd. Stock solution of PFOA (2.42 $\mathrm{mmol} / \mathrm{L})$ in ultrapure water was prepared and stored $\left(4-10^{\circ} \mathrm{C}\right)$. A desired volume of dilute PFOA solution (1.96-3.06 $\mu \mathrm{mol} /$ L) in ultrapure water was prepared for each test using the stock solution.

Irradiation sources and emission intensities

A $20 \mathrm{~W}$ LPUV lamp (UVL20PS-6) and a $110 \mathrm{~W}$ LPUV lamp (EUV110US-94L) manufactured by SEN Light Corporation were used in the tests. Measured emission intensities of major $(254 \mathrm{~nm})$ and minor $(185 \mathrm{~nm})$ wavelengths from the two LPUV lamps are shown in Fig. 1. The $185 \mathrm{~nm}$ intensities for 20 and $110 \mathrm{~W}$ lamps were about 55 and $78 \%$ of the respective 254-nm intensities. The $185 \mathrm{~nm}$ intensity for $110 \mathrm{~W}$ lamp was about fourfold larger than the corresponding intensity for $20 \mathrm{~W}$ lamp. Similarly, the 254- and 185-nm intensities 


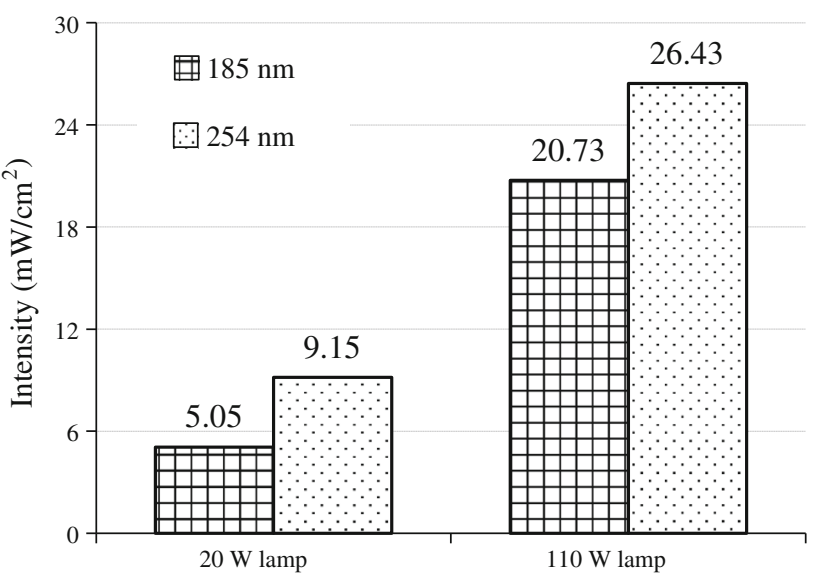

Fig. 1 Emission intensities of low-pressure UV lamps used in the tests

for $110 \mathrm{~W}$ lamp were about 2.9- and 4.1-fold of the corresponding intensities for $20 \mathrm{~W}$ lamp. The increase in 185 -nm intensity for $110 \mathrm{~W}$ lamp is very notable compared to the increase in 254-nm intensity.

\section{Reactor setup and test procedure}

A cylindrical glass reactor (ID: $10 \mathrm{~cm}, \mathrm{H}: 20 \mathrm{~cm}, \mathrm{~V}: 1.5 \mathrm{~L}$ ) described earlier (Giri et al. 2011, 2012) was used for batch tests. A tubular double-layered fused silica glass lamp sleeve with continuous water circulation through the space between the layers was used to ensure transmission of $254 \mathrm{~nm}$ alone to reaction solution. A tubular single-layered synthetic fused silica glass lamp sleeve was used to ensure maximum transmission of $185 \mathrm{~nm}$ in addition to $254 \mathrm{~nm}$ transmission to reaction solution. The lamp sleeves were sealed at the top, and nitrogen gas was continuously passed through the space between lamp and lamp sleeve to remove oxygen and for cooling.

About 1.2 and $1.4 \mathrm{~L}$ of reaction solution were used for the tests with UVC and VUV, respectively. An appropriate volume of diluted PFOA solution was poured into the reactor. The solution was continuously mixed using a magnetic bar and stirrer $(\approx 300 \mathrm{rpm})$. An appropriate volume of freshly prepared KI solution was added to reaction solution (initial $\mathrm{KI}$ concentration $=0.3 \mathrm{mmol} / \mathrm{L}$ ) for reductive degradation tests. The particular KI concentration used in these tests is based on a very similar test conducted earlier with UVC and KI (Qu et al. 2010). Solution $\mathrm{pH}$ and DO in reaction solution were continuously monitored. No solution $\mathrm{pH}$ was adjusted in any of the tests. Pure nitrogen gas $(99.9 \%)$ was purged into the reaction solution for about $30 \mathrm{~min}$ before beginning of the tests for reductive degradation to remove $\mathrm{DO}$, and the purging continued throughout the test period to ensure anoxic condition. All the tests were carried out for $3 \mathrm{~h}$, and samples were taken at selected time intervals for analysis.

Sample analysis

Perfluorooctanoic acid (PFOA) and other short carbonchain perfluoroalkyl carboxylates in samples were measured using liquid chromatography tandem mass spectrometry (LCMS/MS, Applied Biosystems). Chromatographic separation was carried out using ZORBAX Eclipse XDB-C18 column $(2.1 \times 150 \mathrm{~mm}, 3.5 \mu \mathrm{m})$ with $200 \mu \mathrm{L} / \mathrm{min}$ flow rate. Ammonium formate in ultrapure water $(10 \mathrm{mmol} / \mathrm{L})(\mathrm{A})$ and acetonitrile (B) was used as mobile phases. Each measurement lasted for $30 \mathrm{~min}$ with gradient flow of the mobile phases and $5-\mu \mathrm{L}$ sample volume. The eluent gradient started with $90 \%$ of "A" that linearly decreased to $70 \%$ over the first $3 \mathrm{~min}$. Then, it further linearly decreased to $2 \%$ until $20 \mathrm{~min}$, and this was held constant until $25 \mathrm{~min}$. Then, it increased again to $90 \%$ at $25.1 \mathrm{~min}$, held constant until $30 \mathrm{~min}$, and the measurement was terminated. Electrospray ionization (ESI) was the ion source, while multiple reactions monitoring (MRM) method was used for mass scanning. Nitrogen was used as collision and curtain gas (30 psi) and air was used as nebulizer and dryer gas. Ion source voltage and dryer gas temperature were set at $-4,500 \mathrm{mV}$ and $400{ }^{\circ} \mathrm{C}$, respectively. The scanned mass numbers for PFOA, PFHpA, PFHxA, PFPeA, PFBA, PFPrA and TFA were 412.9/368.9, 362.8/319.0, 312.8/268.9, 262.8/218.9, $212.9 / 138.9,162.9 / 119.0$ and $112.9 / 68.9$, respectively. Similarly, the mass numbers for PFOA internal standard were 416.8/371.9. Each of the compounds was quantified using a corresponding six-point linear calibration curve $(0-100 \mu \mathrm{g} / \mathrm{L})$.

Fluoride ion concentrations in the samples were measured using ion chromatography (ICS-2000, Dionex). A main column [IonPac AS20 $(4.0 \times 250 \mathrm{~mm}, 7.5 \mu \mathrm{m})]$ in series with a guard column [IonPac AG20 $(4.0 \times 50 \mathrm{~mm}$, $11 \mu \mathrm{m})]$ was used in chromatographic separation. Potassium hydroxide $(\mathrm{KOH})$ solution was used as eluent with gradient flow rate of $1.0 \mathrm{~mL} / \mathrm{min}$. The column temperature and sample injection volume were $30^{\circ} \mathrm{C}$ and $200 \mu \mathrm{L}$, respectively, while each of the measurements lasted for $25 \mathrm{~min}$. Each measurement started with $5 \mathrm{mmol} / \mathrm{L} \mathrm{KOH}$ concentration in eluent and it remained so until $5 \mathrm{~min}$. The concentration then increased linearly to $30 \mathrm{mmol} / \mathrm{L}$ from 5 to $15 \mathrm{~min}$, and it again increased linearly to $45 \mathrm{mmol} / \mathrm{L}$ from 15 to $25 \mathrm{~min}$ ending the measurement. The fluoride ions quantification was based on a five-point calibration curve $(0-200 \mu \mathrm{g} / \mathrm{L}$ fluoride). Organic carbon concentrations in the samples were measured using a total organic carbon (TOC) analyzer (TOC- $\mathrm{V}_{\mathrm{CSH} / \mathrm{CSN}}$, Shimadzu 
Corporation, Japan) with high-sensitivity catalyst for low TOC concentrations.

\section{Results and discussion}

The PFCs concentrations, removal ratios and rates, and half-life periods mentioned in the following sections refer to water phase only. PFOA removal and defluorination in these tests are best described by the first-order reaction kinetic. Therefore, the first-order reaction rate values for the two processes are used in discussing the results in this section. The terms "UVC" and "VUV" used in this section denote 254-nm wavelength and combined wavelengths (both 254 and $185 \mathrm{~nm}$ ), respectively. Moreover, the numbers " 20 " and " 110 " immediately after "UVC" and "VUV" indicate rated lamp powers. Both photolysis (i.e., photooxidation) and photoreduction tests were conducted with $20 \mathrm{~W}$ lamp, while only photooxidation tests were conducted with $110 \mathrm{~W}$ lamp.

PFOA removal ratios and rates

Figure 2a illustrates PFOA removal profiles in terms of ratio and concentration for the five tested cases. The removal ratio profiles were very similar to the corresponding removal concentration profiles. The 3-h removal ratio values for UVC20, VUV20 and VUV110 were about 31,87 and $100 \%$, respectively. The values for UVC20/KI and VUV20/KI were about 39 and $72 \%$, respectively. The removal ratio for UVC20/KI was $1.3-$ fold of the value for UVC20. On the other hand, the ratio for VUV20/KI was 0.8 -fold of the value for VUV20 for the same reaction period. Unlike with UVC, photoreductive degradation of PFOA with VUV is apparently very inefficient than the corresponding photooxidative degradation. Among the five cases, photooxidation reaction with increased $185 \mathrm{~nm}$ UV energy transmission to reaction solution was the most efficient method for PFOA degradation. For instance, 1-h PFOA removal ratio for VUV110 (97\%) was about 1.6-fold of the value for VUV20 (59\%). In terms of first-order PFOA removal rate (Table 1), UVC20/KI was about $10 \%$ more efficient than UVC20, while VUV20/KI was about $38 \%$ less efficient than VUV20 in these tests. The fourfold increase in $185 \mathrm{~nm}$ UV energy transmission to reaction solution for VUV110 case (Fig. 1) is distinctly reflected on the first-order PFOA removal rate value and half-life period (Table 1).

The PFOA removal rate for UVC20/KI in this investigation is about 2.6-fold larger than the value
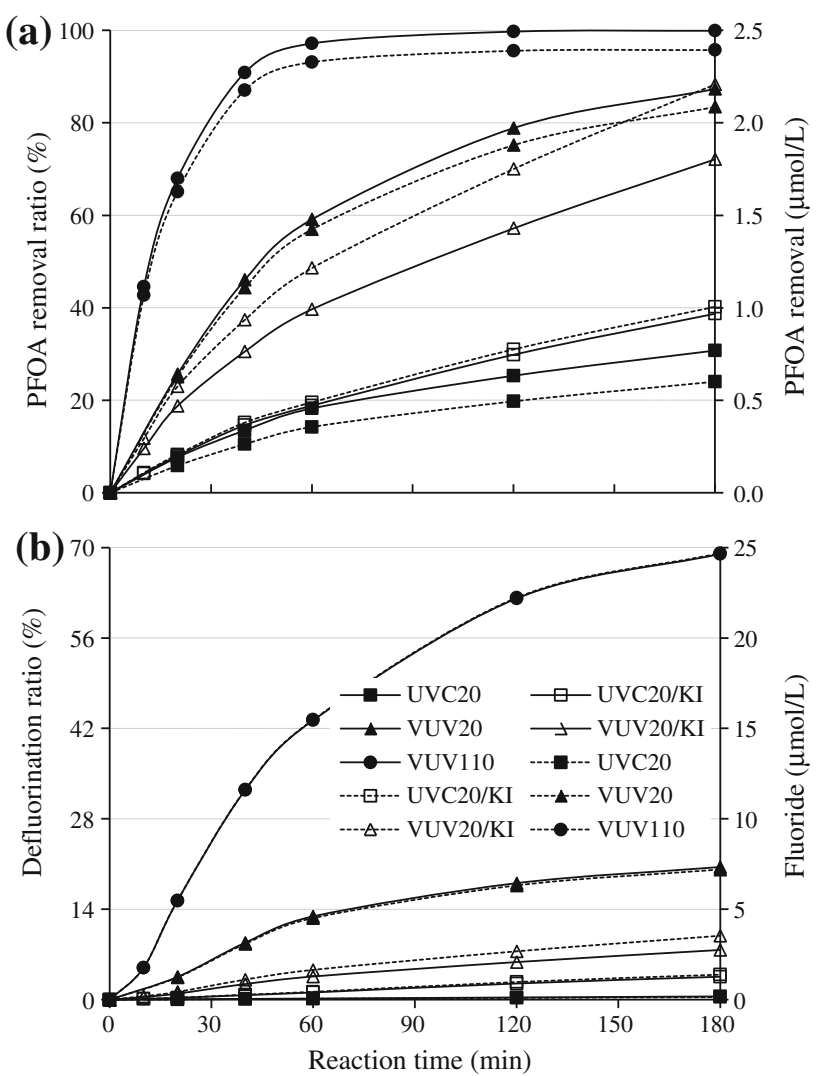

Fig. 2 a PFOA removal and $\mathbf{b}$ defluorination profiles for photooxidation and photoreduction reactions. The solid lines (primary $y$-axis) represent ratios, while the dotted lines (secondary $y$-axis) represent concentrations. The average initial PFOA concentrations in these tests varied between 1.96 and $3.06 \mu \mathrm{mol} / \mathrm{L}$

reported in Park et al. (2009 and 2010), but it is about twofold smaller than the value reported in $\mathrm{Qu}$ et al. (2010). An optimum KI dosage and alkaline solution $\mathrm{pH}$ $(\approx 9.0)$ are shown to enhance reductive degradation of PFOA with UVC (Qu et al. 2010). The twofold smaller value in the present case may be attributed to low solution $\mathrm{pH}$ (initial value $\approx 5.5$, no adjustment/buffering done during the tests) and the choice of KI concentration $(0.3 \mathrm{mmol} / \mathrm{L})$. The very small PFOA removal efficiency with VUV20/KI compared to that for VUV20 may be explained as follows. The reported PFOA degradation rates for photooxidation (Giri et al. 2011, 2012) and photoreduction reactions with aquated electrons $(\mathrm{Qu}$ et al. 2010) are as follows:

$\mathrm{UV}(254 \mathrm{~nm})+\mathrm{PFOA} \rightarrow \mathrm{P}\left(k=17.4 \times 10^{-2} \mathrm{~h}^{-1}\right)$
$\mathrm{UV}(254 \mathrm{~nm}+185 \mathrm{~nm})+\mathrm{PFOA} \rightarrow \mathrm{P}\left(k=81.6 \times 10^{-2} \mathrm{~h}^{-1}\right)$

$e_{\mathrm{aq}}^{-}+\mathrm{PFOA} \rightarrow \mathrm{P}\left(k=43.8 \times 10^{-2} \mathrm{~h}^{-1}\right)$ 
Table 1 First-order rate values and half-life periods

\begin{tabular}{llllll}
\hline Test conditions & UVC20 & UVC20/KI & VUV20 & VUV20/KI & VUV110 \\
\hline PFOA removal & & & & & \\
$k\left(\mathrm{~h}^{-1}\right)$ & $20.2 \times 10^{-2}$ & $22.2 \times 10^{-2}$ & $81.8 \times 10^{-2}$ & $50.4 \times 10^{-2}$ & $357.6 \times 10^{-2}$ \\
$R^{2}$ & 0.9835 & 0.9868 & 0.9803 & 0.9928 & 0.9917 \\
$t_{1 / 2}(\mathrm{~h})$ & 3.50 & 3.12 & 0.85 & 1.37 & 0.19 \\
Defluorination & & & & & \\
$k\left(\mathrm{~h}^{-1}\right)$ & $0.2 \times 10^{-2}$ & $1.2 \times 10^{-2}$ & $14.3 \times 10^{-2}$ & $3.0 \times 10^{-2}$ & $58.8 \times 10^{-2}$ \\
$R^{2}$ & 0.9977 & 0.9952 & 0.9933 & 0.9756 & 0.9958 \\
$t_{1 / 2}(\mathrm{~h})$ & 449.26 & 57.76 & 5.00 & 23.10 & 1.18 \\
\hline
\end{tabular}

where $\mathrm{P}$ denotes degradation products. A rough PFOA photooxidation rate value for $185-\mathrm{nm}$ photons alone can be calculated to be $64.2 \times 10^{-2} \mathrm{~h}^{-1}$ from Eqs. (10) and (12), and this value is about 1.5 -fold larger than the value for aquated electrons [Eq. (12)]. Also, the PFOA removal rate value for VUV20 case in this investigation is about 1.6-fold larger than the value for VUV20/KI case (Table 1). It is evident from both experimental and calculated rate values that photoreductive degradation of PFOA with 185-nm photons and $\mathrm{KI}$ is very inefficient compared to photooxidative degradation with the photons. It appeared that all the available $185-\mathrm{nm}$ photons in the system were utilized for reductive reactions with $\mathrm{KI}$ eliminating any possibility of direct photolysis reaction of PFOA and other short carbonchain PFCs with 185-nm photons. But, the effects (if any) of KI dosage and solution $\mathrm{pH}$ on photoreductive degradation of PFOA with $185-\mathrm{nm}$ photons are yet to be understood.

\section{Defluorination ratios and rates}

Figure $2 b$ shows defluorination profiles in terms of ratio and concentration. The ratio profiles were very similar to the corresponding concentration profiles. Moreover, the defluorination ratio profiles were very similar to PFOA removal ratio profiles (Fig. 2a). However, defluorination ratios were very small than the corresponding PFOA removal ratios. For example, 3 -h defluorination ratio values for UVC20, UVC20/KI, VUV20, VUV20/KI and VUV110 were $0.5,3.5,20.5,7.7$ and $69.0 \%$, respectively. The ratio for UVC20/KI was about sevenfold of the value for UVC20, but the ratio for VUV20 was about 2.7 -fold of the value for VUV20/KI. In terms of first-order defluorination rate values (Table 1), UVC20/ KI was about sixfold more efficient than UVC20, while VUV20/KI was about 4.7-fold inefficient than VUV20. It is to be noted here that the effects of reductive reaction with aquated electrons are very profound on defluorination than on PFOA removal both with UVC and with VUV. Similar to PFOA removal rate, the fourfold increase in 185-nm intensity for VUV110 case is clearly reflected on the defluorination rate and half-life period.

The uniqueness of photoreductive degradation of PFOA with UVC and KI is greatly enhanced defluorination that is attributed to direct cleavage of $\mathrm{C}-\mathrm{F}$ bond by aquated electrons ( $\mathrm{Qu}$ et al. 2010). In this study also, defluorination rate for UVC20/KI increased by sixfold compared to 1.1-fold increase in PFOA removal rate (the values for UVC20 as references). However, defluorination ratio value for UVC20/ $\mathrm{KI}(3.5 \%)$ was very small compared to the value $(\approx 50 \%)$ reported by Qu et al. (2010) for the similar reaction period. The very big discrepancy in these results is attributed to continuous nitrogen gas purging to reaction solution for photoreductive reactions resulting in easy volatilization of short carbon-chain fluorinated intermediates that ultimately escaped out of the reactor. Similar to PFOA removal, the choice of $\mathrm{KI}$ dosage and low solution $\mathrm{pH}$ is thought to be other reasons for the small defluorination with UVC20/KI. Mainly two factors are thought to be responsible for the very low defluorination with VUV20/KI compared to that with VUV20. Despite a slightly different PFOA defluorination mechanisms with UV photooxidation and reduction reactions, the reason mentioned earlier for PFOA removal [Eqs. (1)-(3)] should be valid for defluorination too. Furthermore, losses of short carbon-chain intermediates in gas phases due to continuous nitrogen gas purging are thought to have significantly decreased water phase defluorination for VUV20/ KI case. Similar to PFOA removal, any effects of KI dosage and solution $\mathrm{pH}$ on defluorination for VUV20/KI are yet to be understood. The very big difference in defluorination between VUV20 and VUV20/KI may also be an indication that all of the available $185-\mathrm{nm}$ photons were utilized in generating aquated electrons eliminating any possibility of oxidation reaction with the photons. Understanding reactivity of KI with 254- and 185-nm photons should clarify the point. 
Short carbon-chain fluorinated products and mineralization

Concentration profiles of PFOA and its short carbonchain fluorinated intermediates in water phase during PFOA photodecomposition are illustrated in Fig. 3. The Fig. 3a, b shows VUV20/KI and VUV110 cases, respectively. Though six intermediates at significantly large concentrations were observed with VUV110, only PFHpA, PFHxA, PFPeA and PFBA at low concentrations were observed with VUV20/KI. The concentration profiles of intermediates with VUV20 case (not shown) were very similar, but concentrations were considerably larger than those with VUV20/KI. No other short carbonchain fluorinated intermediates than PFHpA and PFHxA at very low concentrations were observed with UVC20 and UVC20/KI cases. Although the total concentrations of PFOA and its short carbon-chain fluorinated products at the end of 3-h reaction for UVC20/KI and VUV110 cases were apparently very similar, the VUV20/KI case was characterized by very slow degradations of PFOA and the products, while the VUV110 case was characterized by very fast degradations of the compounds. Also, in terms of TOC removals for 3 -h reactions,
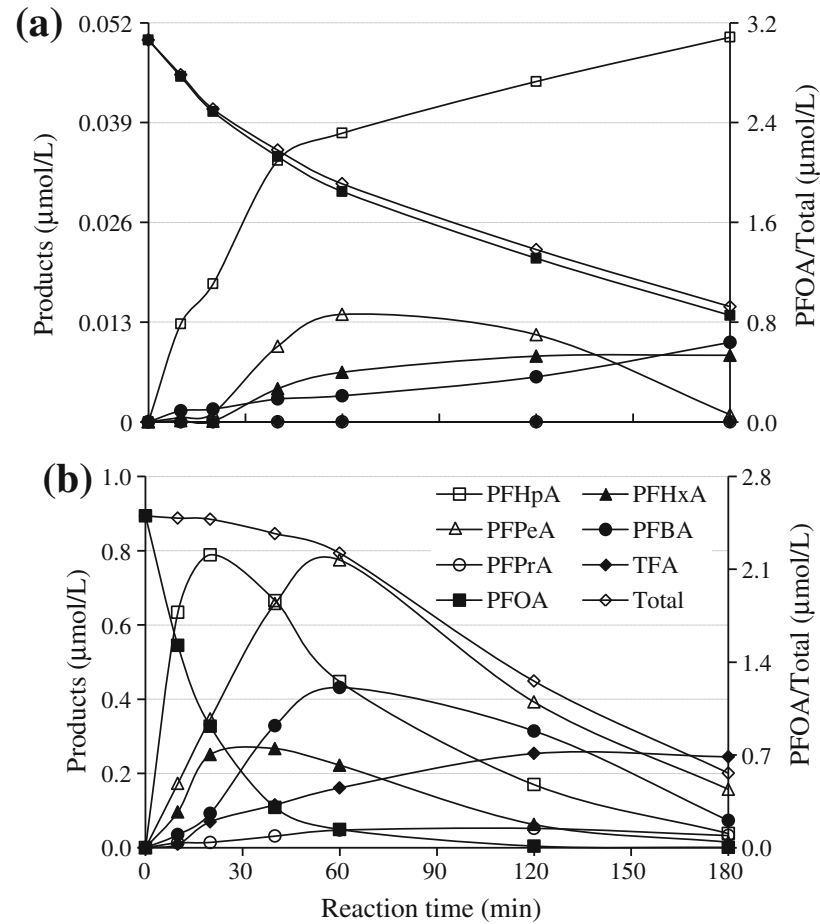

Fig. 3 Concentration profiles of PFOA and its short carbon-chain fluorinated products for a VUV20/KI and b VUV110 cases. The average initial PFOA concentrations in these cases were 3.06 and 2.51 $\mu \mathrm{mol} / \mathrm{L}$, respectively

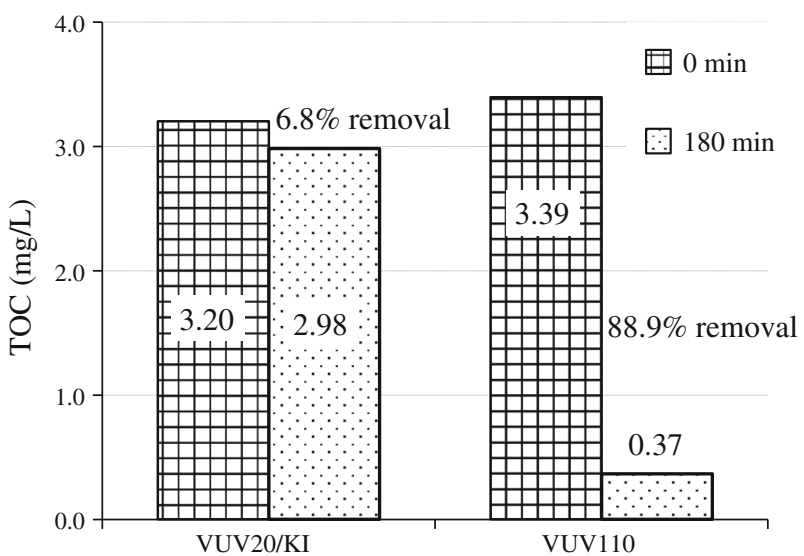

Fig. 4 Measured TOC concentrations at the beginning and end of 180-min reactions for VUV20/KI and VUV110 cases

VUV20/KI was very inefficient ( $\approx 7 \%$ removal, Fig. 4) than VUV20, while VUV110 was the most efficient ( $\approx 89 \%$ removal, Fig. 4) among the five cases.

Similar to PFOA removal and defluorination, the inefficiency of VUV20/KI compared to that of VUV20 for PFOA photodecomposition was clearly observed in the formation of short carbon-chain fluorinated intermediates and TOC removals although no distinction was observed between UVC20 and UVC20/KI in this regard. Unlike in defluorination, any impact of volatilization of fluorinated intermediates on TOC removal particularly with VUV20/ KI was not apparent possibly due to the very low removal efficiency. The fourfold increase in 185-nm photon energy with VUV110 (Fig. 1) mineralized PFOA almost completely (Fig. 4). The considerably small defluorination (69\%) compared to the corresponding TOC removal $(89 \%)$ in this case may partly be attributed to volatilization of short carbon-chain fluorinated intermediates like TFA, PFPrA and PFBA.

UV photolysis of KI with 254- and 185-nm photons

Although formation of aquated electrons due to KI photolysis with $254-$ and $185-\mathrm{nm}$ photons was confirmed in the 1960s (Jortner et al. 1964), their (i.e., $e_{\mathrm{aq}}^{-}$) usefulness in reductive degradation of PFCs was recognized recently (Huang et al. 2007; Park et al. 2009; Park 2010; Qu et al. 2010). Similar to those results, the UVC20/KI process was more efficient than UVC20 in terms of PFOA elimination and defluorination in this investigation. The process efficiencies should greatly be enhanced further at elevated $\mathrm{pH}(\geq 9.0)$ with an optimum KI concentration (if any) for the tested conditions $(\mathrm{Qu}$ et al. 2010). Unlike with UVC20/KI, VUV20/KI was very 
inefficient than VUV20 in terms of PFOA removal, defluorination and short carbon-chain fluorinated intermediates in this investigation as suggested by PFOA photolysis rate with $185-\mathrm{nm}$ photons and PFOA degradation rate with $e_{\mathrm{aq}}^{-}$[Eqs. (1)-(3)]. Moreover, simultaneous occurrence of photolytic degradation of PFOA by $185-\mathrm{nm}$ photons and PFOA degradation by $e_{\mathrm{aq}}^{-}$in presence of both 185- and 254-nm photons in reductive reaction conditions with $\mathrm{KI}$ was not apparent. In this scenario, photolytic reactivity of KI with the two wavelengths should provide clear insight. Jortner et al. (1964) reported large (3.3-fold) quantum yield of $e_{\mathrm{aq}}^{-}$ formation with $185-\mathrm{nm}$ photons than with 254-nm photons in iodide solution. This may be taken as clear evidence that the available $185-\mathrm{nm}$ photon energy was used for the formation of $e_{\mathrm{aq}}^{-}$in VUV20/KI eliminating any possibility of photolysis reaction with the photons ultimately resulting in decreased PFOA mineralization efficiency. Any impacts of solution $\mathrm{pH}$ and $\mathrm{KI}$ concentration in the reactions are yet to be understood. Nevertheless, determination of KI reactivity with 185- and 254-nm photons using today's experimental facilities should be an option for more insight.

\section{Conclusion}

Unlike with UVC, photoreductive mineralization of PFOA in water with VUV was very inefficient than the corresponding photooxidative mineralization, which is attributed to considerably low reactivity of $e_{\mathrm{aq}}^{-}$with PFOA and its fluorinated intermediates than that of $185-\mathrm{nm}$ photons. Photolysis of PFOA and other short carbon-chain PFCs with 185-nm photons in reductive reaction conditions was not apparent due to very high reactivity of $185-\mathrm{nm}$ photons with iodide than that of 254-nm photons. The results showed that 185-nm photons can be best utilized in oxidative degradations, not in reductive degradations. This is the first investigation on photoreductive degradation of PFOA with 185-nm photons that will be a useful reference on VUV usage for degradations of refractory micropollutants.

Acknowledgments This research was carried out under the project "Strategic Research Foundation Grant-Aided Project for Private Universities" (2012-2017) financially supported by the Ministry of Education, Culture, Sports, Science and Technology (MEXT), Japan.

\section{References}

Cao MH, Wang BB, Yu HS, Wang LL, Yuan SH, Chen J (2010) Photochemical decomposition of perfluorooctanoic acid in aqueous periodate with VUV and UV light irradiation. J Hazard Mater 179:1143-1146. doi:10.1016/j.jhazmat.2010.02.030

Chen J, Zhang P (2006) Photodegradation of perfluorooctanoic acid in water under irradiation of $254 \mathrm{~nm}$ light by use of persulfate. Water Sci Technol 54(11-12):317-325. doi:10.21 66/wst.2006.731

Chen J, Zhang P, Liu J (2007) Photodegradation of perfluorooctanoic acid by $185 \mathrm{~nm}$ vacuum ultraviolet light. J Environ Sci 19:387-390. doi:10.1016/S1001-0742(07)60064-3

Clara M, Scheffknecht C, Scharf S, Weiss S, Gans O (2008) Emissions of perfluorinated alkylated substances (PFAS) from point sources: identification of relevant branches. Water Sci Technol 58(1):59-66. doi:10.2166/wst.2008.641

Corsini E, Sangiovanni E, Avogadro A, Galbiati V, Viviani B, Marinovich M, Galli CL, Dell'Agli M, Germolec DR (2012) In vitro characterization of the immunotoxic potential of several perfluorinated compounds (PFCs). Toxicol Appl Pharmacol 258:248-255. doi:10.1016/j.taap.2011.11.004

Domingo JL (2012) Health risks of dietary exposure to perfluorinated compounds. Environ Int 40:187-195. doi:10.1016/j.envint. 2011.08.001

Giri RR, Ozaki H, Morigaki T, Taniguchi S, Takanami R (2011) UV photolysis of perfluorioctanoic acid (PFOA) in dilute aqueous solution. Water Sci Technol 63(2):276-282. doi:10.2166/ wst.2011.050

Giri RR, Ozaki H, Okada T, Taniguchi S, Takanami R (2012) Factors influencing UV photodecomposition of perfluoroocanoic acid in water. Chem Eng J 180:197-203. doi:10.1016/j. cej.2011.11.049

Huang L, Dong W, Hou H (2007) Investigation of the reactivity of hydrated electron toward perfluorinated carboxylates by laser flash photolysis. Chem Phys Lett 436:124-128. doi:10.1016/j. cplett.2007.01.037

Iglev H, Trifonov A, Thaller A, Vuchvarov I, Fiebig T, Laubereau A (2005) Photoionization dynamics of an aqueous iodide solution: the temperature dependence. Chem Phys Lett 403:198-204. doi: 10.1016/j.cplett.2005.01.014

Jortner J, Ottolenghi M, Stein G (1964) On the photochemistry of aqueous solutions of chloride, bromide and iodide. J Phys Chem 68(2):247-255. doi:10.1021/j100784a005

Lau C, Butenhoff JL, Rogers JM (2004) The developmental toxicity of perfluoroalkyl acids and their derivatives. Toxicol Appl Pharmacol 198:231-241. doi:10.1016/j.taap.2003.11.031

Lien NPH, Fuji S, Tanaka S, Nozoe M, Wirojanagud W, Anton A, Lindstrom G (2006) Perfluorinated substances in tap water of Japan and several countries and their relationship to surface water contamination. Environ Eng Res 43:611-618. http:// sciencelinks.jp/j-east/article/200704/000020070407A0046125. php. Accessed 15 May 2012

Park H (2010) Photolysis of aqueous perfluorooctanoate and perfluorooctane sulfonate. Rev Roum Chim 55(10):611-619. http:// revroum.getion.ro/wp-content/uploads/2010/RRCh_10_2010/ Art\%2002.pdf. Accessed 12 July 2012

Park H, Vecitis CD, Cheng J, Choi W, Mader BT, Hoffman MR (2009) Reductive defluorination of aqueous perfluorinated alkyl surfactants: effects of ionic head group and chain length. J Phys Chem A 113:690-696. doi:10.1021/jp807116q

Qu Y, Zhang C, Li F, Chen J, Zhou Q (2010) Photo-reductive defluorination of perfluorooctanoic acid in water. Water Res 44:2939-2947. doi:10.1016/j.watres.2010.02.019

Saito N, Harada K, Inoue K, Sasaki K, Yoshinaga T, Koizumi A (2004) Perfluorooctonate and perfluorooctane sulfonate concentrations in surface water in Japan. J Occup Health 46:49-59. 
https://www.jstage.jst.go.jp/article/joh/46/1/46_1_49/_pdf. Accessed 26 June 2012

Tang H, Xiang Q, Lei M, Yan J, Zhu L, Zou J (2012) Efficient degradation of perfluorooctanoic acid by UV-Fenton process. Chem Eng J 184:156-162. doi:10.1016/j.cej.2012.01.020
Wang Y, Zhang P, Pan G, Chen H (2008) Ferric ion mediated photochemical decomposition of perfluorooctanoic acid (PFOA) by $254 \mathrm{~nm}$ UV light. J Hazard Mater 160:181-186. doi: 10.1016/j.jhazmat.2008.02.105 